\title{
PERFIL FíSICO-QUímICO E NUTRICIONAL DE CARNE DE FRANGO DE CRIAÇÃO ORGÂNICA DISPONÍVEL NO COMÉRCIO VAREJISTA
}

\author{
LUCIANE SILVIA ROSSA ${ }^{* *}$ \\ EVELYN VON ROSEN STAHLKE* \\ MINEIA ALESSANDRA SCARANELLO MALAQUIAS ${ }^{2 * *}$ \\ DEISY CRISTINA DIEZ* \\ SÔNIA CACHOEIRA STERTZ $3^{3 * *}$ \\ RENATA ERNLUND FREITAS DE MACEDO ${ }^{4 * * * *}$
}

\begin{abstract}
O objetivo deste trabalho foi avaliar as características físico-químicas e nutricionais de carne de frango orgânico disponível no comércio varejista. Foram analisadas 25 carcaças de frango orgânico e 25 de frango convencional, sendo de cinco marcas diferentes em cada grupo. Foram realizadas as determinações de $\mathrm{pH}$, cor instrumental, drip test, composição centesimal, perda de peso pelo cozimento, força de cisalhamento, teor de colesterol e perfil de ácidos graxos. A comparação dos resultados entre as diferentes marcas dentro de cada sistema de criação foi realizada por ANOVA e teste de Tukey $(p<0,05)$. A comparação dos resultados entre o grupo orgânico e o convencional foi realizada pelo teste $t(p<0,05)$. Para a maioria dos parâmetros analisados, as carnes de ambos os sistemas de criação mostraram-se semelhantes. Houve diferença para $\mathrm{pH}$, perda de peso pelo cozimento, teor de gordura, colesterol, cinzas e cor instrumental $(p<0,05)$. A carne orgânica obteve maior rendimento durante o cozimento e maior valor de $a^{*}$ (cor vermelha) tanto na forma crua quanto após cozimento. A carne orgânica apresentou maior teor de gordura $(p<0,05)$, porém ainda é considerada magra, visto que o teor foi inferior a $1 \%$ e apresentou menor teor de colesterol $(p<0,05)$. Na comparação de marcas comerciais, verificou-se maior variação de resultados $(p<0,05)$ entre as marcas de frango orgânico, mostrando menor uniformidade nos atributos de qualidade físico-química entre as carcaças produzidas nesse sistema, o que pode dificultar seu reconhecimento como um produto de segmento específico de mercado pelo consumidor no país.
\end{abstract}

PALAVRAS-CHAVE: AVICULTURA ORGÂNICA; CARNE; QUALIDADE.

1 * Alunas do curso de Mestrado em Ciência Animal, Escola de Ciências Agrárias e Medicina Veterinária, Pontifícia Universidade Católica do Paraná (PUCPR), São José dos Pinhais (PR). E-mail: lu.rossa@hotmail.com, sarvinhh@hotmail.com, evelyn.stahlke@gmail.com

2 Alunas do curso de Medicina Veterinária, Escola de Ciências Agrárias e Medicina Veterinária, PUCPR, São José dos Pinhais (PR). E-mail: mineia.marini@hotmail.com, deisyeti@hotmail.com

3 Pesquisadora Científica do Departamento de Engenharia Química, Universidade Federal do Paraná (UFPR), Curitiba (PR). E-mail: stertz@ufpr.br

$4 \quad$ Docente do curso de Mestrado em Ciência Animal, Escola de Ciências Agrárias e Medicina Veterinária, PUCPR, São José dos Pinhais (PR). Autor para correspondência: BR 376, km 14, CEP 83010-500, São José dos Pinhais (PR). Fone: (41) 3299-4334, fax: (41) 3299-4423. E-mail: renata.macedo@pucpr.br 


\section{INTRODUÇÃO}

Os produtos orgânicos são muito valorizados no mercado internacional, o que pode ser verificado pelo rápido crescimento da agricultura orgânica mundial nas últimas décadas (MICHAELIDOU; HASSAN, 2010). O aumento do consumo de produtos orgânicos é decorrente do maior interesse dos consumidores por uma alimentação mais saudável, natural e equilibrada, sem adição de produtos químicos sintéticos, e que preserve o meio ambiente, visto que na produção orgânica não se permite o emprego de produtos químicos ou substâncias que possam gerar resíduos no produto final (POUTA et al., 2010).

Devido ao crescimento do consumo de alimentos orgânicos, o estudo da sua qualidade e segurança também desperta interesse da comunidade científica, assim como os possíveis efeitos benéficos que a sua ingestão pode trazer à saúde do consumidor (REMBIAŁKOWSKA; ŚREDNICKA, 2009).

A agricultura orgânica surgiu no Brasil na década de 1970; porém, a expansão do sistema de produção ocorreu a partir dos anos 1980 com o crescimento da conscientização da preservação ecológica (CUNHA et al., 2010). Apesar disso, a experiência com a produção orgânica/agroecológica no Brasil ainda é recente e pequena e concentrada em alimentos de origem vegetal, como frutas e hortaliças. Por isso, não há muitos dados sobre a qualidade desses alimentos, especialmente no que se refere a carnes orgânicas (FIGUEIREDO, 2010).

Face à demanda crescente por produtos orgânicos no Brasil, atualmente esses produtos são encontrados para comércio tanto em grandes redes varejistas, ou em mercados exclusivos de produtos orgânicos, como nas tradicionais feiras, onde são vendidos diretamente do produtor.

Fanatico et al. (2005) relataram que nos Estados Unidos, onde os alimentos orgânicos são mais conhecidos e consumidos pela população, verifica-se aumento na demanda de produtos avícolas orgânicos. No Brasil, a produção de carne de frango orgânico ainda é uma atividade recente, ainda que essa seja o tipo de carne orgânica mais disponível aos consumidores do país.

Embora o mercado de alimentos orgânicos se apoie na alegação de que esses alimentos possuem melhor qualidade nutricional e são mais seguros que os alimentos convencionais, ainda não há dados científicos que comprovem essas alegações nos alimentos que estão disponíveis no comércio varejista e que são adquiridos e consumidos pela população brasileira.

Nesse sentido, este trabalho teve como objetivo avaliar a qualidade físico-química e nutricional de carne de frango orgânico ofertada no comércio varejista aos consumidores brasileiros.

\section{MATERIAL E MÉTODOS}

Inicialmente, o presente trabalho pretendia avaliar a qualidade da carne de frango orgânico disponível no comércio varejista da região de Curitiba (PR), visto que essa cidade foi a primeira no país a inaugurar um mercado municipal exclusivo para o comércio de produtos orgânicos. Contudo, após a pesquisa de mercado, foram encontradas apenas duas marcas certificadas de carne de frango orgânico disponíveis no comércio da cidade. Dessa forma, optou-se por ampliar os locais de aquisição das amostras para que se obtivesse um número maior de marcas/fornecedores avaliados.

Após a busca por marcas de frango orgânico em outras cidades e estados do país, foi possível encontrar no comércio varejista e feiras livres cinco diferentes marcas comerciais certificadas de frango orgânico. Foram adquiridas 25 carcaças de frango orgânico, inteiras e congeladas sendo cinco de cada marca comercial, de produtores dos estados do Espírito Santo, Paraná, Santa Catarina e São Paulo e certificados por instituição reconhecida no país para produção orgânica -, e processadas em frigoríficos com Serviço de Inspeção Federal ou Estadual. Informações sobre o manejo de criação orgânica dos animais, raças utilizadas e idade ao abate foram obtidas diretamente com os produtores mediante entrevista. 
As amostras foram mantidas congeladas a $-18^{\circ} \mathrm{C}$ durante a aquisição e o transporte ao local de análise. Após a chegada ao laboratório, as carcaças foram pesadas em balança semianalítica (Gehaka, BK 3000, SP, Brasil) e avaliadas sobre absorção de água pelo drip test seguindo as recomendações da legislação brasileira (BRASIL, 1998). Após esse teste, o corte de peito, sem pele, foi desossado para a realização das demais determinações.

$\mathrm{O}$ pH foi determinado por pHmetro digital com eletrodo de penetração (Quimis, São Paulo, Brasil) nas porções musculares do peito em cinco replicatas (AOAC, 2000). A perda de peso pelo cozimento foi realizada de acordo com a metodologia de Froning e Uijtteenboogaart (1988) em porções de peito embaladas em saco plástico e cozidas por imersão em água até atingir temperatura interna de $82^{\circ} \mathrm{C}$. A determinação instrumental de cor foi realizada com colorímetro portátil (Minolta CR-410, Japan) acionado diretamente sobre a superfície externa do peito cru e após cozimento, em cinco replicatas, utilizando as coordenadas $L^{*}, a^{*}, b^{*}$ do sistema CIELab. A força de cisalhamento foi determinada em porções retangulares $(1 \mathrm{~cm}$ de largura $\times 1 \mathrm{~cm}$ de altura $\times 2 \mathrm{~cm}$ de comprimento) de peito cozido conforme descrito anteriormente e cisalhadas, em cinco replicatas, em texturômetro TA-XT2i Texture Analyzer (Stable Micro Systems, Surrey, UK) equipado com lâmina Warner Bratzler (FRONING; UIJTTEENBOOGAART, 1988).

$O$ perfil de ácidos graxos foi determinado nas amostras inicialmente saponificadas com hidróxido de potássio e metanol 0,5 M segundo metodologia de Hartman e Lago (1973). A quantificação de ácidos graxos foi realizada na porção sobrenadante em cromatógrafo a gás (CP 3900, Varian, CA, USA) equipado com detector FID, injetor split na razão de 1:100 e coluna cromatográfica CPSIL 88. O gás de arraste utilizado foi o nitrogênio. Utilizou-se o software Varian Star Workstation 5.0 para tratamento dos dados.

O teor de colesterol foi determinado seguindo metodologia de Al-Hasani et al. (1993), com a preparação das amostras pela saponificação dos lipídeos em solução alcoólica de hidróxido de potássio seguida de extração dos insaponificáveis e determinação de colesterol em cromatógrafo líquido (HP 1100, Agilent Technologies, CA, USA).

Ainda que as condições exatas de manejo de criação, abate e processamento das carcaças de frango sejam desconhecidas, pelo fato de terem sido adquiridas no comércio varejista, como o objetivo deste estudo foi avaliar a carne de frango orgânico que o consumidor adquire no comércio varejista, e levando-se em consideração a alegação de que o alimento orgânico possui qualidade superior à do convencional, foram também analisadas 25 carcaças de frango produzidas no sistema convencional e processadas em frigoríficos com Serviço de Inspeção Federal do Distrito Federal, Mato Grosso, Paraná e Santa Catarina. As condições de aquisição, transporte, armazenamento e procedimentos de análise das carcaças convencionais foram iguais às empregadas para as carcaças orgânicas.

Os resultados das diferentes marcas de frango orgânico foram analisados por ANOVA e teste de Tukey para comparação das médias. Os resultados das marcas de frango convencional também foram comparados entre si por ANOVA e teste de Tukey.

Os dados obtidos em cada um dos grupos (orgânico e convencional) foram comparados pelo teste $t$, sendo desconsideradas as diferenças entre as marcas dentro de cada grupo. Em todas as análises foi adotado o nível de significância de 5\% e o software GraphPad Prism version 3.00 para Windows (CA, USA).

\section{RESULTADOS E DISCUSSÃO}

As carcaças de frango orgânico avaliadas pertenciam às raças/linhagens Cobb, Label Rouge (Pescoço Pelado) e Embrapa 041. Os produtores informaram que os frangos orgânicos foram abatidos com idades entre 49 e 106 dias (média de 77,5 dias), conforme apresentado na Tabela 1. Com relação à dieta nutricional dos animais, os produtores relataram utilizar alimentação orgânica à base de milho, farelo de soja, farelo de trigo e também farinha de ostra e farinha de 
peixe, porém não especificaram suas proporções na dieta. Para os frangos de criação convencional, não foram coletados dados relativos à idade de abate ou raça, visto que o produto industrial é bastante padronizado no mercado brasileiro, com idade de abate variando entre 42 e 45 dias e uso de linhagens comerciais.

TABELA 1. RAÇA E IDADE DE ABATE DOS FRANGOS ORGÂNICOS AVALIADOS

\begin{tabular}{ccc}
\hline Marca & Raça & Idade de Abate \\
\hline OA & Cobb & 49 dias \\
\hline OB & Label Rouge (Pescoço pelado) & 94 dias \\
\hline OC & Embrapa 041 & 85 dias \\
\hline OD & Cobb & 60 dias \\
\hline OE & Label Rouge (Pescoço pelado) & 106 dias \\
\hline
\end{tabular}

A variação de idade de abate verificada para o grupo orgânico deve-se ao fato de que na produção orgânica de frango no Brasil podem ser utilizadas raças de linhagem caipira ou industrial, que possuem diferentes velocidades de crescimento (BRASIL, 2008). Segundo a legislação brasileira, os frangos de linhagem caipira, para serem comercializados com essa designação, deverão ser abatidos somente após 85 dias de idade (BRASIL, 1999). Por outro lado, os frangos de linhagem industrial - de crescimento rápido - que são criados em sistemas orgânicos não poderiam ser abatidos com essa mesma idade, devido ao risco de desenvolverem enfermidades decorrentes do peso muito elevado em relação aos animais de linhagem caipira (ZANUSSO; DIONELLO, 2003).

O peso médio das carcaças de frango orgânico foi de $2,098 \mathrm{~kg}$, e o das carcaças de frango convencional, $2,256 \mathrm{~kg}$, não havendo diferença significativa $(p>0,05)$ entre os grupos, mas com diferenças entre as marcas dentro de cada grupo $(p<0,05)$, como mostra a Tabela 2.

As diferentes idades de abate dos frangos das marcas orgânicas pode justificar a grande diferença de peso entre as carcaças desse sistema.

\section{TABELA 2. PESO MÉDIO DAS CARCAÇAS DE FRANGOS AVALIADAS, SEPARADOS EM GRUPO ORGÂNICO E CONVENCIONAL ( $\mathrm{N}=50$ AMOSTRAS)}

\begin{tabular}{ccccccc}
\hline \multirow{2}{*}{$\begin{array}{c}\text { Peso } \\
\text { médio } \\
\text { das } \\
\text { carcaças } \\
(\mathbf{k g})\end{array}$} & $\mathrm{OA}$ & $\mathrm{OB}$ & $\mathrm{OC}$ & $\mathrm{OD}$ & $\mathrm{OE}$ & Orgânico \\
\cline { 2 - 7 } & $1,856^{\mathrm{b}} \pm 0,21$ & $1,671^{\mathrm{b}} \pm 0,12$ & $1,813^{\mathrm{b}} \pm 0,19$ & $3,502^{\mathrm{a}} \pm 0,10$ & $1,647^{\mathrm{b}} \pm 0,08$ & $2,098^{\mathrm{A}} \pm 0,73$ \\
\cline { 2 - 7 } & $1,883^{\mathrm{c}} \pm 0,05$ & $2,548^{\mathrm{a}} \pm 0,11$ & $2,283^{\mathrm{b}} \pm 0,11$ & $2,337^{\mathrm{ab}} \pm 0,18$ & $2,231^{\mathrm{b}} \pm 0,07$ & $2,256^{\mathrm{A}} \pm 0,25$ \\
\hline
\end{tabular}

$\mathrm{OA}$ a $\mathrm{OE}=$ marcas de frango orgânico. $\mathrm{CA}$ a $\mathrm{CE}=$ marcas de frango convencional. Valores médios com letras minúsculas diferentes na mesma linha são significativamente diferentes em nível de $5 \%$ dentro de cada grupo (orgânico e convencional). Valores com letras maiúsculas diferentes na mesma coluna indicam diferença significativa em nível de $5 \%$ entre o grupo orgânico e convencional. 
O valor médio de pH para a carne de peito do grupo orgânico foi 5,88 e 6,07 para o grupo convencional, havendo diferença significativa $(p<0,05)$ entre os dois grupos (Tabela 3). $\mathrm{O} p \mathrm{H}$ apresentou-se dentro da faixa entre 5,5 e 6,3, citada por Shimokomaki et al. (2006) para filés de peito de frango. Corroborando os resultados do presente estudo, Castellini et al. (2002) também observaram maiores valores de $\mathrm{pH}$ em carne de peito de frango convencional $(\mathrm{pH} 5,96)$ em relação à de frango orgânico $(\mathrm{pH} 5,80)(\mathrm{p}<0,05)$, sugerindo que o menor valor de $\mathrm{pH}$ da carne orgânica poderia estar relacionado às melhores condições de bem-estar desses animais, que diminui o estresse pré-abate e preserva as reservas de glicogênio muscular, causando maior redução de pH post mortem.

TABELA 3. VALORES DE PH, DRIP TEST E PERDA DE PESO POR COZIMENTO (\%) NAS CARCAÇAS DE FRANGO ORGÂNICO CONVENCIONAL AVALIADAS ( $\mathrm{N}=50$ AMOSTRAS)

\begin{tabular}{|c|c|c|c|}
\hline \multirow{2}{*}{ Marcas } & $\mathrm{pH}$ & $\begin{array}{c}\text { Absorção de água \% (Drip } \\
\text { test) }\end{array}$ & $\begin{array}{c}\text { Perda de peso no cozimento } \\
(\%)\end{array}$ \\
\hline & Média \pm DP & Média \pm DP & Média \pm DP \\
\hline \multicolumn{4}{|c|}{ Marcas de frango orgânico } \\
\hline OA & $5,56^{b} \pm 0,32$ & $6,52^{b} \pm 2,02$ & $22,07^{\mathrm{ab}} \pm 2,16$ \\
\hline OB & $5,96^{a} \pm 0,21$ & $6,52^{b} \pm 0,93$ & $17,58^{c} \pm 1,93$ \\
\hline OC & $5,99^{a} \pm 0,08$ & $10,29^{a} \pm 1,07$ & $18,29^{b} \pm 2,07$ \\
\hline OD & $5,89^{\mathrm{ab}} \pm 0,03$ & $2,63^{c} \pm 0,83$ & $25,04^{a} \pm 1,78$ \\
\hline OE & $6,00^{\mathrm{a}} \pm 0,16$ & $2,56^{c} \pm 1,50$ & $21,89^{\mathrm{ab}} \pm 2,35$ \\
\hline Orgânico & $5,88^{\mathrm{B}} \pm 0,24$ & $5,70^{A} \pm 3,19$ & $20,98^{B} \pm 3,36$ \\
\hline \multicolumn{4}{|c|}{ Marcas de frango convencional } \\
\hline CA & $6,03^{a} \pm 0,20$ & $6,37^{a} \pm 0,79$ & $23,17^{a} \pm 1,67$ \\
\hline $\mathrm{CB}$ & $6,07^{\mathrm{a}} \pm 0,11$ & $6,04^{\mathrm{ab}} \pm 1,21$ & $26,13^{a} \pm 3,40$ \\
\hline $\mathrm{CC}$ & $6,18^{\mathrm{a}} \pm 0,12$ & $5,02^{\mathrm{ab}} \pm 0,71$ & $25,92^{\mathrm{a}} \pm 2,42$ \\
\hline CD & $6,03^{\mathrm{a}} \pm 0,07$ & $5,79^{\mathrm{ab}} \pm 1,08$ & $22,52^{\mathrm{a}} \pm 1,58$ \\
\hline CE & $6,00^{\mathrm{a}} \pm 0,15$ & $4,63^{b} \pm 0,50$ & $23,32^{a} \pm 1,37$ \\
\hline Convencional & $6,07^{\mathrm{A}} \pm 0,14$ & $5,57^{A} \pm 1,05$ & $24,21^{\mathrm{A}} \pm 2,54$ \\
\hline
\end{tabular}

$\mathrm{OA}$ a $\mathrm{OE}=$ marcas de frango orgânico. $\mathrm{CA}$ a $\mathrm{CE}=$ marcas de frango convencional. $\mathrm{DP}=$ desvio padrão. Valores médios com letras minúsculas diferentes na mesma coluna são significativamente diferentes em nível de $5 \%$ dentro de cada grupo (orgânico e convencional). Valores com letras maiúsculas diferentes na mesma coluna indicam diferença significativa em nível de $5 \%$ entre as médias do grupo orgânico e convencional. 
A perda média de peso verificada pelo drip test nas carcaças de frango orgânico foi semelhante à obtida para as carcaças de frango convencional (5,70\% e 5,57\%, respectivamente), conforme visto na Tabela 3. O drip test avalia a quantidade de água perdida pelo descongelamento das carcaças de frango, sendo permitido o valor máximo de 6\% (BRASIL, 1998). Apesar de o valor médio para o drip test ter sido abaixo de 6\%, das 25 carcaças de frango orgânico 12 (48\%) apresentaram perda superior ao limite permitido, atingindo valores de até $10,29 \%$. Para o grupo convencional, das 25 amostras, nove (36\%) apresentaram teor de água acima do permitido. Um estudo realizado pelo Instituto de Defesa do Consumidor em 2004 constatou que de oito marcas de frango convencional avaliadas no país, sete apresentavam valores de drip test acima do limite permitido.

Para a perda de peso pelo cozimento (PPC), foram obtidos valores de $20,98 \%$ para carne de frango orgânico e de $24,21 \%$ para a carne convencional $(p<0,05)$, conforme apresentado na Tabela 3. A capacidade de retenção de água da carne e as perdas de peso pelos processos de frigorificação, congelamento e cozimento tendem a ser maiores em valores mais próximos ao ponto isoelétrico das proteínas da carne, pH 5,3 a 5,5 (SHIMOKOMAKI et al., 2006). Apesar de ter apresentado menor valor de $\mathrm{pH}$, a capacidade de retenção de água durante o cozimento da carne orgânica não se mostrou comprometida. Resultados semelhantes foram observados por Husak et al. (2008) em carne de frango caipira, com menor valor de pH em relação à carne convencional, porém com maior rendimento no cozimento. Contrariando esses resultados, Castellini et al. (2002) encontraram maior PPC em peito de frango orgânico $(33,45 \%)$ em relação ao convencional $(31,10 \%)$. Faria et al. (2009) também obtiveram PPC para carne de frango caipira abatido aos 85 dias $(23,46 \%)$ superior à verificada neste trabalho.

A menor perda de peso pelo cozimento proporciona à carne de frango orgânico maior rendimento em processamentos ou preparações culinárias com tratamento térmico ou que exijam alta capacidade de retenção de água, sendo de interesse na industrialização de derivados cozidos ou marinados.

A maciez é outro atributo de importância para a qualidade da carne e pode ser afetada pela perda de peso durante o cozimento. Ela está diretamente relacionada com a satisfação final do consumidor e pode ser medida instrumentalmente pela força de cisalhamento. Para esse parâmetro, os valores médios da carne orgânica $(2,70 \mathrm{~kg})$ foram semelhantes aos da carne convencional $(2,18$ kg) $(p>0,05)$.

Aguiar (2006) relata maior força de cisalhamento para peito de frango caipira (1,87 kgf) quando comparado a frango convencional (1,83 kgf). Contreras-Castillo et al. (2007) avaliaram a força de cisalhamento de peito de frango alternativo e encontraram valores de 2,67 a 3,45 kgf. Lima (2005) observou menor força de cisalhamento em amostras de peito de frango convencional $(1,97$ kgf) quando comparado a peito de frango caipira (2,46 kgf). A maior força de cisalhamento em peito de frango orgânico ou caipira em comparação ao convencional relatada pelos autores foi justificada pela maior idade de abate e maior exercício muscular dos animais criados em sistema livre, visto que a maturidade do tecido conjuntivo e o volume das fibras musculares aumentam a força de cisalhamento da carne.

A cor da carne é um importante atributo de qualidade para os consumidores, tanto para a seleção da carne fresca no varejo quanto para avaliação final e aceitação no momento do consumo (QIAO et al., 2001). Na avaliação da cor instrumental, verificou-se diferença significativa $(p<0,05)$ entre o grupo orgânico e o convencional para a luminosidade $\left(L^{*}\right)$ e intensidade de cor vermelha $\left(a^{*}\right)$ da carne crua de peito. Na avaliação da carne cozida, houve diferença significativa $(p<0,05)$ entre os grupos somente para a intensidade de cor vermelha $\left(a^{*}\right)$, conforme visto na Tabela 4. 


\section{TABELA 4. VALORES DE LUMINOSIDADE (L*), COR VERMELHA (A*) E AMARELA (B*) PARA O PEITO DE FRANGO ORGÂNICO E CONVENCIONAL CRU E COZIDO ( $\mathbf{N}=50$ AMOSTRAS)}

\begin{tabular}{|c|c|c|c|c|c|c|}
\hline \multirow{2}{*}{ Amostras } & \multicolumn{2}{|c|}{$\begin{array}{c}L^{*} \\
\text { Média } \pm \text { DP }\end{array}$} & \multicolumn{2}{|c|}{$\begin{array}{c}a^{*} \\
\text { Média } \pm \mathrm{DP} \\
\end{array}$} & \multicolumn{2}{|c|}{$\begin{array}{c}b^{*} \\
\text { Média } \pm \mathrm{DP}\end{array}$} \\
\hline & Carne crua & Carne cozida & Carne crua & Carne cozida & Carne crua & Carne cozida \\
\hline \multicolumn{7}{|c|}{ Marcas de frango orgânico } \\
\hline OA & $\begin{array}{c}63,84^{\mathrm{ab}} \pm \\
1,54\end{array}$ & $\begin{array}{c}69,33^{\mathrm{bc}} \pm \\
3,94\end{array}$ & $13,09^{a} \pm 1,63$ & $2,17^{a} \pm 0,53$ & $15,85^{a} \pm 1,66$ & $\begin{array}{c}12,65^{\mathrm{ab}} \pm \\
0,43\end{array}$ \\
\hline OB & $63,83^{b} \pm 1,53$ & $\begin{array}{c}73,73^{\mathrm{ab}} \pm \\
3,98\end{array}$ & $10,65^{b} \pm 1,09$ & $2,22^{\mathrm{a}} \pm 0,25$ & $12,12^{b} \pm 1,40$ & $11,94^{\mathrm{b}} \pm 0,87$ \\
\hline OC & $67,72^{\mathrm{a}} \pm 2,43$ & $65,74^{c} \pm 3,34$ & $9,74^{b} \pm 0,59$ & $3,23^{\mathrm{a}} \pm 0,77$ & $10,26^{b} \pm 0,97$ & $\begin{array}{c}12,79^{\mathrm{ab}} \pm \\
0,88\end{array}$ \\
\hline OD & $61,97^{b} \pm 2,37$ & $\begin{array}{c}71,65^{\mathrm{bc}} \pm \\
2,30\end{array}$ & $\begin{array}{c}11,33^{\mathrm{ab}} \pm \\
0,93\end{array}$ & $2,47^{a} \pm 0,35$ & $15,80^{\mathrm{a}} \pm 2,09$ & $13,73^{a} \pm 0,84$ \\
\hline OE & $61,22^{b} \pm 2,17$ & $77,82^{\mathrm{a}} \pm 1,78$ & $10,84^{b} \pm 1,17$ & $2,79^{a} \pm 0,98$ & $12,49^{b} \pm 2,06$ & $\begin{array}{c}12,78^{\mathrm{ab}} \pm \\
1,23 \\
\end{array}$ \\
\hline Orgânico & $63,72^{\mathrm{B}} \pm 2,96$ & $71,65^{\mathrm{A}} \pm 5,07$ & $11,13^{\mathrm{A}} \pm 1,53$ & $2,58^{A} \pm 0,70$ & $13,30^{A} \pm 2,72$ & $12,78^{\mathrm{A}} \pm 1,00$ \\
\hline \multicolumn{7}{|c|}{ Marcas de frango convencional } \\
\hline CA & $69,52^{\mathrm{a}} \pm 1,70$ & $70,73^{b} \pm 1,38$ & $7,97^{\mathrm{b}} \pm 0,37$ & $2,23^{\mathrm{ab}} \pm 0,18$ & $8,47^{\mathrm{b}} \pm 1,32$ & $11,36^{\mathrm{b}} \pm 0,82$ \\
\hline CB & $\begin{array}{c}66,88^{\mathrm{ab}} \pm \\
3,42\end{array}$ & $68,23^{b} \pm 3,20$ & $11,08^{a} \pm 0,85$ & $1,99^{\mathrm{ab}} \pm 0,14$ & $14,96^{a} \pm 0,43$ & $11,47^{b} \pm 0,66$ \\
\hline CC & $69,42^{\mathrm{a}} \pm 2,87$ & $70,20^{b} \pm 0,99$ & $10,15^{\mathrm{a}} \pm 1,36$ & $1,69^{b} \pm 0,39$ & $16,35^{\mathrm{a}} \pm 2,61$ & $11,16^{\mathrm{b}} \pm 0,50$ \\
\hline$C D$ & $63,70^{b} \pm 1,55$ & $78,79^{a} \pm 2,87$ & $10,77^{a} \pm 1,35$ & $2,54^{a} \pm 0,61$ & $13,61^{a} \pm 0,97$ & $14,15^{\mathrm{a}} \pm 1,27$ \\
\hline CE & $\begin{array}{c}65,52^{\mathrm{ab}} \pm \\
1,45 \\
\end{array}$ & $81,98^{a} \pm 1,22$ & $10,46^{a} \pm 0,81$ & $1,92^{\mathrm{ab}} \pm 0,39$ & $15,01^{a} \pm 1,18$ & $15,75^{a} \pm 1,10$ \\
\hline Convencional & $67,01^{A} \pm 3,14$ & $73,99^{A} \pm 5,83$ & $10,08^{B} \pm 1,46$ & $2,07^{\mathrm{B}} \pm 0,46$ & $13,68^{\mathrm{A}} \pm 3,11$ & $12,78^{A} \pm 2,06$ \\
\hline
\end{tabular}

$\mathrm{OA}$ a $\mathrm{OE}=$ marcas de frango orgânico. $\mathrm{CA}$ a $\mathrm{CE}=$ marcas de frango convencional. $\mathrm{DP}=$ desvio padrão. $\mathrm{L}^{*}=$ luminosidade, $a^{*}=$ vermelho, $b^{*}=$ amarelo. Valores médios com letras minúsculas diferentes na mesma coluna são significativamente diferentes em nível de $5 \%$ dentro de cada grupo (orgânico e convencional). Valores com letras maiúsculas diferentes na mesma coluna indicam diferença significativa em nível de $5 \%$ entre as médias do grupo orgânico e convencional.

A maior intensidade de cor vermelha observada para o peito dos frangos orgânicos pode ser explicada pela maior atividade física exercida por esses animais pelo acesso a ambiente externo de criação. Além disso, a idade dos animais também pode ser um fator que afeta a intensidade da cor vermelha da carne. A carne de aves mais velhas apresentam maiores concentrações de mioglobina quando comparada à carne de aves mais jovens (HUSAK et al., 2008). Tendo em vista que os frangos orgânicos avaliados neste trabalho foram abatidos em idade superior à dos frangos convencionais, a maior intensidade de cor vermelha pode estar relacionada à maior concentração de mioglobina na carne.

Para a carne cozida de peito das linhagens caipiras Pescoço Pelado e Paraíso Pedrês, Faria et al. (2009) citam valores inferiores aos obtidos neste trabalho para $L^{*}(47,70)$ e $b^{*}(5,70)$ e superiores para $a^{*}(5,10)$. Por sua vez, Castellini et al. (2002) encontraram valores inferiores para os três parâmetros de cor em amostras cozidas de peito de frango orgânico.

Em ambos os grupos avaliados observou-se aumento dos valores de luminosidade e redução da intensidade de cor vermelha e amarela após o cozimento do peito. A cor vermelha foi o parâmetro de maior variação antes e após o cozimento, o que pode estar associado à desnaturação térmica da mioglobina presente na carne durante o cozimento, que causa mudança em sua coloração, tornando-a menos vermelha (KING; WHYTE, 2006). 
O teor de umidade e proteínas mostrou-se semelhante na carne de frangos orgânicos e convencionais. Porém, para os teores de lipídeos e cinzas foram observadas diferenças significativas $(p<0,05)$ entre os grupos (Tabela 5).

Os valores de umidade obtidos para carne orgânica $(73,40 \%)$ e convencional $(73,55 \%)$ foram ligeiramente inferiores aos verificados por Husak et al. (2008), que encontraram teor de umidade de $74,98 \%$ em peito de frango orgânico e $75,52 \%$ em peito de frango convencional, porém dentro da faixa estabelecida pela legislação brasileira para o teor de umidade em corte de peito de frango, resfriado ou congelado e sem pele, que deve estar entre 73,36\% e 75,84\% (BRASIL, 2010).

TABELA 5. COMPOSIÇÃO CENTESIMAL DA CARNE DE PEITO DE FRANGO DE CRIAÇÃO ORGÂNICA E CONVENCIONAL (N = 50 AMOSTRAS)

\begin{tabular}{|c|c|c|c|c|c|c|}
\hline Marcas & $\begin{array}{l}\text { Umidade } \\
(\mathrm{g} / 100 \mathrm{~g})\end{array}$ & $\begin{array}{l}\text { Proteínas } \\
(\mathrm{g} / 100 \mathrm{~g})\end{array}$ & $\begin{array}{l}\text { Lipídeos } \\
\text { (g/100g) }\end{array}$ & $\begin{array}{l}\text { Cinzas } \\
(\mathrm{g} / 100 \mathrm{~g})\end{array}$ & $\begin{array}{c}\text { Cálcio } \\
\text { (mg/100g) }\end{array}$ & $\begin{array}{l}\text { Fósforo } \\
\text { (g/100g) }\end{array}$ \\
\hline & Média \pm DP & Média \pm DP & $\begin{array}{c}\text { Média } \pm \\
\text { DP }\end{array}$ & $\begin{array}{c}\text { Média } \pm \\
\text { DP }\end{array}$ & Média \pm DP & Média \pm DP \\
\hline \multicolumn{7}{|c|}{ Marcas de frango orgânico } \\
\hline OA & $71,66^{b} \pm 0,89$ & $\begin{array}{c}22,88^{\mathrm{b}} \pm \\
0,38\end{array}$ & $0,92^{\mathrm{a}} \pm 0,22$ & $\begin{array}{c}0,99^{\mathrm{ab}} \pm \\
0,08\end{array}$ & $0,03^{a}$ & $0,21^{\mathrm{ab}} \pm 0,01$ \\
\hline OB & $72,81^{b} \pm 0,60$ & $\begin{array}{c}23,47^{\mathrm{ab}} \pm \\
0,46\end{array}$ & $0,81^{a} \pm 0,11$ & $\begin{array}{c}1,00^{\mathrm{ab}} \pm \\
0,14\end{array}$ & $0,03^{a}$ & $0,20^{\mathrm{b}} \pm 0,01$ \\
\hline OC & $74,24^{\mathrm{a}} \pm 0,98$ & $\begin{array}{c}23,85^{\mathrm{a}} \pm \\
0,77\end{array}$ & $0,67^{a} \pm 0,23$ & $0,91^{b} \pm 0,03$ & $0,03^{a}$ & $0,21^{\mathrm{ab}} \pm 0,01$ \\
\hline OD & $73,50^{\mathrm{a}} \pm 0,79$ & $\begin{array}{c}23,71^{\mathrm{ab}} \pm \\
0,51\end{array}$ & $0,97^{\mathrm{a}} \pm 0,07$ & $\begin{array}{c}1,09^{\mathrm{a}} \pm \\
0,06^{\mathrm{a}}\end{array}$ & $0,03^{a}$ & $0,22^{\mathrm{a}} \pm 0,01$ \\
\hline OE & $74,81^{\mathrm{a}} \pm 0,70$ & $\begin{array}{c}23,40^{\mathrm{ab}} \pm \\
0,30\end{array}$ & $0,70^{\mathrm{a}} \pm 0,10$ & $\begin{array}{c}0,93^{\mathrm{ab}} \pm \\
0,06\end{array}$ & $0,03^{a}$ & $0,21^{\mathrm{ab}} \pm 0,01$ \\
\hline Orgânico & $73,40^{A} \pm 1,34$ & $\begin{array}{c}23,46^{A} \pm \\
0,58\end{array}$ & $0,82^{\mathrm{A}} \pm 0,19$ & $0,98^{\mathrm{B}} \pm 0,10$ & $0,03^{A}$ & $0,21^{\mathrm{B}} \pm 0,01$ \\
\hline \multicolumn{7}{|c|}{ Marcas de frango convencional } \\
\hline CA & $\begin{array}{c}73,29^{b} \pm \\
0,87\end{array}$ & $\begin{array}{c}22,32^{\mathrm{b}} \pm \\
0,86\end{array}$ & $0,45^{b} \pm 0,25$ & $1,18^{a} \pm 0,11$ & $0,03^{a}$ & $0,21^{a} \pm 0,01$ \\
\hline CB & $\begin{array}{c}72,63^{\mathrm{b}} \pm \\
0,35\end{array}$ & $\begin{array}{c}24,09^{\mathrm{a}} \pm \\
0,47\end{array}$ & $0,78^{\mathrm{a}} \pm 0,08$ & $1,13^{\mathrm{a}} \pm 0,07$ & $0,03^{a}$ & $0,22^{a} \pm 0,01$ \\
\hline CC & $\begin{array}{c}73,55^{\mathrm{ab}} \pm \\
0,41\end{array}$ & $\begin{array}{c}23,98^{\mathrm{a}} \pm \\
0,54\end{array}$ & $0,71^{\mathrm{ab}} \pm 0,06$ & $1,09^{\mathrm{a}} \pm 0,08$ & $0,03^{a}$ & $0,21^{a} \pm 0,01$ \\
\hline CD & $\begin{array}{c}73,16^{\mathrm{b}} \pm \\
0,69\end{array}$ & $\begin{array}{c}24,23^{\mathrm{a}} \pm \\
0,21\end{array}$ & $0,43^{\mathrm{b}} \pm 0,18$ & $1,12^{\mathrm{a}} \pm 0,11$ & $0,03^{a}$ & $0,22^{a} \pm 0,01$ \\
\hline CE & $\begin{array}{c}75,09^{a} \pm \\
0,50\end{array}$ & $\begin{array}{c}22,70^{\mathrm{b}} \pm \\
0,41\end{array}$ & $0,57^{\mathrm{ab}} \pm 0,13$ & $1,20^{\mathrm{a}} \pm 0,08$ & $0,03^{a}$ & $0,22^{a} \pm 0,01$ \\
\hline Convencional & $\begin{array}{c}73,55^{A} \pm \\
1,01\end{array}$ & $\begin{array}{c}23,47^{A} \pm \\
0,95\end{array}$ & $0,59^{\mathrm{B}} \pm 0,20$ & $1,14^{\mathrm{A}} \pm 0,09$ & $0,03^{A}$ & $0,22^{A} \pm 0,01$ \\
\hline
\end{tabular}

$\mathrm{OA}$ a $\mathrm{OE}=$ marcas de frango orgânico. $\mathrm{CA}$ a $\mathrm{CE}=$ marcas de frango convencional. $\mathrm{DP}$ = desvio padrão. Valores médios com letras minúsculas diferentes na mesma coluna são significativamente diferentes em nível de $5 \%$ dentro de cada grupo (orgânico e convencional). Valores com letras maiúsculas diferentes na mesma coluna indicam diferença significativa em nível de $5 \%$ entre as médias do grupo orgânico e convencional. 
Para o teor de proteínas, foram obtidos valores médios de $23,46 \%$ e $23,47 \%$ para carne orgânica e convencional, respectivamente. Esses valores foram ligeiramente superiores aos encontrados por Husak et al. (2008) em peito de frango orgânico $(23,31 \%)$ e em peito de frango convencional (22,26\%), e semelhantes aos descritos por Vieira et al. (2007) para peito de frango da linhagem Cobb (23,76\%). O teor proteico do peito tanto das carcaças orgânicas quanto das convencionais se mostrou em conformidade com o estabelecido pela legislação brasileira para peito de frango, resfriado ou congelado e sem pele, entre $21,05 \%$ e $24,37 \%$ (BRASIL, 2010).

Os teores de lipídeos encontrados para o corte de peito de ambos os grupos de todas as marcas avaliadas foram inferiores a $1 \%$, com variação de $0,67 \%$ a $0,97 \%$ no grupo orgânico e $0,43 \%$ a $0,78 \%$ no grupo convencional. Apesar de ser considerada carne magra, o teor médio de lipídeos em peito do grupo orgânico foi superior ao do grupo convencional $(p<0,05)$. A maior deposição de lipídeos nas carcaças orgânicas pode estar relacionada à maior idade de abate desses animais.

Para o teor de cinzas, foi observada diferença significativa $(p<0,05)$ entre os dois grupos, sendo que os valores médios obtidos para frango convencional foram maiores que os de frango orgânico. Segundo a Tabela Brasileira de Composição dos Alimentos, o peito de frango convencional sem pele apresenta média de cinzas de $1 \mathrm{~g} / 100 \mathrm{~g}$ (TACO, 2006). Esse valor assemelhou-se mais ao valor médio obtido em frangos orgânicos $(0,98 \%)$ do que em frangos convencionais $(1,14 \%)$.

Os teores médios de ácidos graxos saturados, monoinsaturados e poli-insaturados na carne de peito dos frangos orgânicos e convencionais não apresentaram diferença significativa ( $p>0,05)$. Nos grupos orgânico e convencional, os ácidos graxos saturados $(39,10 \%$ e $39,98 \%$, respectivamente) e os monoinsaturados $(40,84 \%$ e $40,19 \%$, respectivamente) foram predominantes, com pequena diferença entre seus teores totais. Os ácidos graxos poli-insaturados representaram $14,59 \%$ e $14,46 \%$ dos ácidos graxos na carne desses grupos, respectivamente.

Entre os ácidos saturados, o teor de ácido palmítico foi semelhante $(p>0,05)$ nas amostras orgânicas e convencionais $(30,60 \%$ e $30,22 \%$, respectivamente). Porém, para o ácido esteárico verificou-se teor superior $(p<0,05)$ em carne convencional $(9,76 \%)$ em relação à carne orgânica $(8,50 \%)$. Os ácidos graxos mais comumente encontrados em carnes são palmítico (C16:0), esteárico (C18:0) e oleico (C18:1) (VALSTA et al., 2005).

Entre os ácidos monoinsaturados, que representaram a maior proporção dos ácidos graxos presentes, o ácido oleico foi o mais abundante, com proporções de $36,67 \%$ na carne orgânica e $36,34 \%$ na convencional. Os ácidos graxos monoinsaturados apresentam efeitos benéficos à saúde pela prevenção de doenças cardiovasculares e redução das concentrações plasmáticas de LDL (lipoproteínas de baixa densidade) em indivíduos normais. Dietas ricas em ácidos graxos monoinsaturados e antioxidantes foram associadas ao baixo estresse oxidativo e à redução da oxidação de LDL (LAPOINTE et al., 2006).

Os teores de ácidos monoinsaturados observados em carne de ambos os grupos foram superiores aos relatados por Husak et al. (2008), que obtiveram 31,67\% em peito de frango orgânico e $39,13 \%$ em frango convencional adquiridos no comércio varejista da região Centro-Oeste dos EUA.

No que se refere ao teor de ácidos poli-insaturados, foram observados valores inferiores aos obtidos por Husak et al. (2008) tanto para a carne orgânica quanto convencional $(38,19 \%$ para frango orgânico e 28,57\% para convencional). Essas diferenças podem ocorrer devido a diferenças na dieta alimentar dos animais, visto que a composição de ácidos graxos na dieta afeta a proporção de gordura e o perfil lipídico da carcaça dos frangos (DU et al., 2000). Não foi observada a presença de ácidos graxos trans nas amostras de peito de ambos os grupos. Os ácidos graxos trans aumentam a concentração plasmática do LDL e diminuem a concentração do HDL (lipoproteína de alta densidade), estando diretamente relacionados ao risco de doenças coronarianas (VIEIRA, 2007).

Para o colesterol, verificou-se teor superior $(p<0,05)$ em peito de frango convencional $(73,29 \mathrm{mg} / 100 \mathrm{~g})$ em comparação ao orgânico $(61,58 \mathrm{mg} / 100 \mathrm{~g})$. Esses valores foram superiores ao descrito na Tabela Brasileira de Composição dos Alimentos (TACO, 2006) para peito de frango sem pele (59 mg/100 g) e aos encontrados por Aguiar (2006) para peito de frango caipira 
$(48,6 \mathrm{mg} / 100 \mathrm{~g})$ e convencional $(58,40 \mathrm{mg} / 100 \mathrm{~g})$, porém inferiores aos descritos pelo USDA (1990) para carne de frango sem pele $(80 \mathrm{mg} / 100 \mathrm{~g})$.

Para a maioria dos parâmetros físico-químicos analisados, as carnes de frango orgânico e convencional foram semelhantes. Por isso, é provável que a escolha dos consumidores por produtos orgânicos envolva outros aspectos além dos parâmetros físico-químicos analisados nas amostras avaliadas neste estudo. Entre as poucas diferenças observadas, a cor da carne orgânica é provavelmente o atributo de maior impacto na decisão de escolha do consumidor, pois ela é determinante para a compra de carne.

\section{CONCLUSÃO}

Do ponto de vista físico-químico e nutricional, para a maioria dos parâmetros avaliados a carne de frango orgânico apresentou composição e perfil semelhantes aos da carne de frango convencional. A menor uniformidade das carcaças e maior variação na qualidade físico-química das diferentes marcas orgânicas poderá dificultar a criação de identidade para a carne de frango orgânico no mercado brasileiro e seu reconhecimento como um produto de segmento específico de mercado pelo consumidor.

\section{ABSTRAT}

\section{PHYSICAL CHEMICAL AND NUTRITIONAL PROFILE OF ORGANIC RETAIL CHICKEN MEAT}

The aim of this study was to evaluate physicochemical and nutritional characteristics of meat from organic chicken available at retail. Carcasses of 25 organic chicken and 25 conventional chicken were analyzed, 5 different brands in each group. Determinations of $\mathrm{pH}$, instrumental color, drip test, chemical composition, cooking loss, shearing force, cholesterol content and fatty acid profile were performed. The comparison of results among different brands within each group (organic or conventional) was performed by ANOVA and Tukey test $(P<0.05)$. The comparison of results between organic and conventional group was performed by $t$ test $(P<0.05)$. For most parameters, the chicken meat of both rearing systems did not show significant differences. Groups showed differences in $\mathrm{pH}$, cooking loss, fat, cholesterol and ash content and instrumental color $(P<0.05)$. Organic chicken obtained higher yield during cooking and higher values of $\mathrm{a}^{*}$ both in raw and cooked meat. Organic meat showed higher fat content $(P<0.05)$, but could still be considered lean, since the content was less than $1 \%$, and showed lower cholesterol content $(P<0.05)$. Organic carcasses of different brands showed greater variation in attributes of physical and chemical quality $(P<0.05)$, which reveals that organic chicken at retail are lesser standardized than conventional chicken. It might hinder the recognition of organic chicken as a product of specific market segment by the Brazilian consumers.

KEYWORDS: FREE RANGE POULTRY, MEAT, QUALITY.

\section{REFERÊNCIAS}

1 AGUIAR, A.P.S. Opinião do consumidor e qualidade da carne de frangos criados em diferentes sistemas de produção. 2006. 70 f. Dissertação (Mestrado em Ciências) - Universidade de São Paulo, Piracicaba, 2006.

2 AL-HASANI, S.M.; HLAVAC, J.; CARPENTER, M.W. Rapid determination of cholesterol in single and multicomponent prepared foods. Journal of AOAC International, v.76, n.4, p.902-906, 1993. 
4 BRASIL. Ministério da Agricultura, Pecuária e Abastecimento. Portaria $\mathrm{n}^{\circ} 210$ de 10 de novembro de 1998. Regulamento técnico da inspeção tecnológica e higiênico-sanitária de carne de aves. Diário Oficial da União, Brasília, DF, 26 nov. 1998.

5 BRASIL. Ministério da Agricultura, Pecuária e Abastecimento. Departamento de Inspeção de Produtos de Origem Animal. Ofício circular DOI/ DIPOA $n^{\circ} 007 / 99$. Registro do produto "frango caipira ou frango colonial" ou "frango tipo ou estilo caipira" ou "tipo ou estilo colonial”. Diário Oficial da União, Brasília, DF, 19 de maio de 1999.

6 BRASIL. Ministério da Agricultura, Pecuária e Abastecimento. Instrução Normativa nº 64, de 18 de dezembro de 2008. Aprova o regulamento técnico para os sistemas orgânicos de produção animal e vegetal. Diário Oficial da União, Brasília, DF, p. 21, 19 dez. 2008.

7 BRASIL. Ministério da Agricultura, Pecuária e Abastecimento. Instrução Normativa n 12, de 26 de Julho de 2010. Estabelece os parâmetros para avaliação do teor total de água contida nos cortes de frangos, resfriados e congelados, na forma dos anexos I e II à presente Instrução Normativa. Diário Oficial da União, Brasília, DF, 27 jul. 2010.

8 CASTELLINI, C.; MUGNAI, C.; DAL BOSCO, A. Effect of organic production system on broiler carcass and meat quality. Meat Science, v.60, n.3, p.219-225, 2002.

9 CONTRERAS-CASTILLO, C.; PINTO, A. A.; SOUZA, G. L.; BERAQUET, N. J.; AGUIAR, A. P.; CIPOLLI, K. M. V. A. B.; MENDES, C. M. I.; ORTEGA, E. M. Effects of feed withdrawal periods on carcass yield and breast meat quality of chickens reared using an alternative system. Poultry Science, v.16, n.4, p.613-622, 2007.

10 DU, M.; AHN, D.U.; SELL, J.L. Effects of dietary conjugated linoleic and linoleic: linolenic acid ratio on polyunsaturated fatty acid status in laying hens. Poultry Science, v.79, n.12, p.1749-1756, 2000.

11 FANATICO, A. C.; CAVITT, L. C.; PILLAI, P. B.; EMMERT, J. L.; OWENS, C. M. Evaluation of slower-growing broiler genotypes grown with and without outdoor access: meat quality. Poultry Science, v.84, n.11, p.1785-1790, 2005.

12 FARIA, P. B.; BRESSAN, M. C.; SOUZA, X. R.; RODRIGUES, É. C.; CARDOSO, G. P.; GAMA, L. T. Composição proximal e qualidade da carne de frangos das linhagens Paraíso Pedrês e Pescoço Pelado. Revista Brasileira de Zootecnia, v.38, n.12, p.2455-2464, 2009.

13 FIGUEIREDO, E. A. P. Produção agroecológica de frangos de corte. 2010. Disponível em: http://frangoc.blogspot. com/2010/04/producao-agroecologica-de-frangos-de.html. Acesso em: 13 nov. 2013.

14 FRONING, G.W.; UIJTTENBOOGAART, T.G. Effect of post mortem electrical stimulation on color, texture, $\mathrm{pH}$, and cooking loses of hot and cold deboned chicken broiler breast meat. Poultry Science, v.67, n.11, p.1536-1544, 1988.

15 HARTMAN, L.; LAGO, R. C. Rapid preparation of fatty acid methyl esters. Laboratory Practices, v.22, n.6, p.475-477, 1973.

16 HUSAK, R.L.; SEBRANEK, J.G.; BREGENDAHL, K. A survey of commercially available broilers marketed as organic, free-range, and conventional broilers for cooked meat yields, meat composition, and relative value. Poultry Science, v.87, n.11, p.2367-2376, 2008 .

17 INSTITUTO BRASILEIRO DE DEFESA DO CONSUMIDOR, [IDEC]. 2004. Teste comparativo - frangos congelados. Disponível em: <http://www2.camara.gov.br/atividade-legislativa/comissoes/comissoes-permanentes/capadr/ audiencias-2008/rap270508idec.pdf> Acesso em: 02/03/2014.

18 KING, N.J.; WHYTE, R. Does it look cooked? A review of factors that influence cooked meat color. Journal of Food Science, v.71, n.4, p.31-40, 2006.

19 LAPOINTE, A.; COUILLARD, C.; LEMIEUX, S. Effects of dietary factors on oxidation of low-density lipoprotein particles. Journal of Nutritional Biochemistry, v.17, n.10, p.645-658, 2006.

20 LIMA, A. M. C. Avaliação de dois sistemas de produção de frango de corte: uma visão multidisciplinar. 2005. $111 \mathrm{f}$. Tese (Doutorado em Engenharia Agrícola) - Faculdade de Engenharia Agrícola, Universidade Estadual de Campinas, 2005.

21 MICHAELIDOU, N.; HASSAN, L. M. Modeling the factors affecting rural consumers' purchase of organic and free-range produce: A case study of consumers' from the Island of Arran in Scotland, UK. Food Policy, v.35, n.2, p.130-139, 2010.

22 POUTA, E.; HEIKKILÄ, J.; FORSMAN-HUGG, S.; ISONIEMI, M.; MÄKELÄ, J. Consumer choice of broiler meat: The effects of country of origin and production methods. Food Quality and Preference, v.21, n.5, p.539-546, 2010.

23 QIAO, M.; FLECTHER, D. L.; SMITH, D. P.; NORTHCUTT, J. K. The effect of broiler breast meat color on pH, moisture, water-holding capacity, and emulsification capacity. Poultry Science, v.80, n.5, p.676-680, 2001.

24 REMBIAŁKOWSKA, E.; ŚREDNICKA, D. Organic food quality and impact on human health. Agronomy Research, v.7, n.2, p.719-727, 2009.

25 SHIMOKOMAKI, M.; OLIVO, R.; TERRA, N. N.; FRANCO, B. D. G. M. Atualidades em ciência e tecnologia de carnes. São Paulo: Livraria Varela, 2006. 236p.

26 TACO. Tabela Brasileira de Composição dos Alimentos. 2006. NEPA-UNICAMP. Versão II. Campinas, SP. Disponível em: <http://www.unicamp.br/nepa/taco/contar/taco_versao2.pdf.> Acesso em: 03/02/2014. 
27 USDA. U.S. DEPARTMENT OF AGRICULTURE. Agricultural Research Service. Composition of foods: beef products, raw, processed, prepared. Agricultural Handbook, Washington DC.: U.S. Government Printing Office, 1990, n.8-13, p.6.

28 VALSTA, L.M.; TAPANAINEN, H.; MÄNNISTÖ, S. Meat fats in nutrition. Meat Science, v.70, n.3, p.525-530, 2005.

29 VIEIRA, J. O.; BRESSAN, M. C.; FARIA, P. B.; FERREIRA, M. W.; FERRÃO, S. P. B.; SOUZA, X. R. Efeito dos métodos de cocção na composição centesimal e colesterol do peito de frangos de diferentes linhagens. Ciência e Agrotecnologia, v.31, n.1, p.164-170, 2007.

30 ZANUSSO, J. T.; DIONELLO, N. J. L. Produção avícola alternativa - Análise dos fatores qualitativos da carne de frangos de corte tipo caipira. Revista Brasileira de Agrociência, v.9, n.3, p. 191-194, 2003. 